\title{
LETTER \\ Channel-Gain-Based Transmission Scheduling for Cooperative Relaying
}

\author{
Naotaka SHIBATA $^{\dagger a}$, Koji YAMAMOTO ${ }^{\dagger \dagger \dagger}$, Members, Hidekazu MURATA $^{\dagger}$, Senior Member, \\ and Susumu YOSHIDA ${ }^{\dagger}$, Fellow
}

\begin{abstract}
SUMMARY A cooperative relaying system with transmission scheduling is investigated. Cooperative relaying is composed of multiple links because the source sends the data to more than one receiver, and the destination receives multiple data transmitted by more than one transmitter. Therefore, if the source can transmit the data when the channel gains of the links are high, it is not clear which channel gains should be high in order to achieve high spectral efficiency. In the present letter, the spectral efficiency of a cooperative relaying system is theoretically derived under the assumption that the source transmits the data only when the channel gains of links are above certain threshold values. Numerical results reveal that a high spectral efficiency can be achieved by assuring a high channel gain for the link with the highest average received power among links to the destination.

key words: cooperative relaying, transmission scheduling, spectral effciency
\end{abstract}

\section{Introduction}

Cooperative relaying is a promising technique for exploiting transmit diversity in a multi-hop transmission [1]-[3]. Diversity gain can be obtained by combining multiple signals at receivers. Therefore, the introduction of this technique can improve the bit error rate or the spectral efficiency (SE), as compared to single-hop or multi-hop transmissions [1], [2]. Moreover, cooperative relaying can improve the total SE of wireless systems while assuring the required throughput for each route [3].

In a number of wireless systems, transmission scheduling (TS) is introduced in order to achieve more efficient use of wireless resources [4]-[6]. By introducing TS, which allows source with good channel conditions to transmit the data, the use of power and frequency can be improved.

However, the introduction of TS into a cooperative relaying system has not yet been reported. Unlike multi-hop transmission, cooperative relaying uses multiple links because the source sends the data to more than one receiver, and the destination receives multiple data transmitted by more than one transmitter. Therefore, if a source can transmit the data when the channel gains of the links are high, which channel gains should be high in order to achieve high $\mathrm{SE}$ is not clear.

Manuscript received March 16, 2009.

Manuscript revised October 31, 2009.

$\dagger$ The authors are with the Graduate School of Informatics, Kyoto University, Kyoto-shi, 606-8501 Japan.

${ }^{\dagger \dagger}$ The author is with Royal Institute of Technology Stockholm, 16440 Sweden.

a) E-mail: contact-h21@ @anase.kuee.kyoto-u.ac.jp

DOI: 10.1587/transcom.E93.B.776
In the present letter, the $\mathrm{SE}$ of a cooperative relaying system is theoretically derived under the assumption that the source can send the data only when the channel gains of links are above certain threshold values. The numerical results of the evaluation suggest that the channel gains of certain specific links should be used in TS in order to achieve high SE.

\section{System Model}

In the present letter, cooperative relaying is assumed to consist of a source, a destination, and a relay station as in [1], [2]. Figure 1 shows the scheme of cooperative relaying investigated in the present letter. In order to realize this scheme, the available time is divided into two time slots of equal size. In slot 1 , the source sends the data to both the relay station and the destination. In slot 2 , the relay station decodes and forwards the data received from the source. A Rayleigh block-fading channel, as described in [1], is assumed.

The SE of the wireless system is evaluated in an interference-limited environment, where the thermal noise is negligible compared to the interference. We approximate the interference signals as additive Gaussian noise, as described in [7]. All sources in the system transmit the data using cooperative relaying, as in [3]. Moreover, all sources are assumed to transmit the data in $100 p_{\text {th }} \%$ of the time by introducing the TS, where $p_{\text {th }}$ represents the transmission probability. These assumptions are reasonable because the goal is to evaluate the effect of introducing the TS to the cooperative relaying system. The transmit power is assumed to be the same at each transmitter. Omnidirectional antennas are used at all stations. There is sufficient distance between any two routes, so that the average interference power is assumed to be the same for all of the transmitters in a given route.

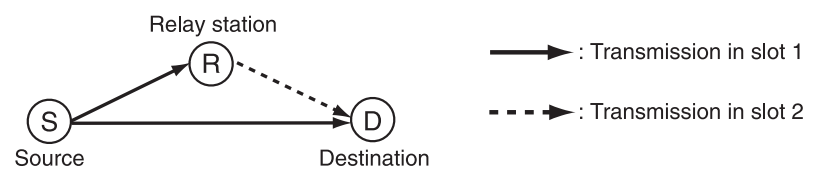

Fig. 1 Scheme of cooperative relaying investigated in the present letter. 


\section{$2.1 \quad \mathrm{TS}$}

In the present analysis, the TS based on channel gains, as described in [4], is adopted. The source is assumed to send data only when the square of the channel gain, $|h|^{2}$, is above a threshold value, $H_{\text {th }}$. The probability $P\left(|h|^{2}>H_{\text {th }}\right)$ that $|h|^{2}$ is higher than $H_{\mathrm{th}}$ is given as

$$
P\left(|h|^{2}>H_{\text {th }}\right)=\int_{H_{\text {th }}}^{\infty} \mathrm{e}^{-x} \mathrm{~d} x=\mathrm{e}^{-H_{\text {th }}} .
$$

This is because the value of $|h|^{2}$ follows an exponential distribution under a Rayleigh fading channel.

\subsection{Cooperative Relaying with TS}

Cooperative relaying consists of three links: the sourcedestination link, the source-relay link, and the relaydestination link. Let us denote the channel gains of the source-destination link, the source-relay link, and the relaydestination link as $h_{\mathrm{SD}}, h_{\mathrm{SR}}$, and $h_{\mathrm{RD}}$, respectively. The TS allows the source to transmit the data only when $h_{\mathrm{SD}}>H_{\mathrm{SD}}$, $h_{\mathrm{SR}}>H_{\mathrm{SR}}$, and $h_{\mathrm{RD}}>H_{\mathrm{RD}}$. Here, $H_{\mathrm{SD}}, H_{\mathrm{SR}}$, and $H_{\mathrm{RD}}$ are the thresholds of the source-destination link, the sourcerelay link, and the relay-destination link, respectively, in TS. For example, if only the source-destination link and the source-relay link are used for TS, then $H_{\mathrm{RD}}$ is set to zero so that the source can transmit the data at any $h_{\mathrm{RD}}$.

These thresholds should be set to satisfy the following relationship for a given transmission time, $p_{\text {th }}$.

$$
\begin{aligned}
p_{\text {th }} & =P\left(\left|h_{\mathrm{SD}}\right|^{2} \geq H_{\mathrm{SD}}\right) \cdot P\left(\left|h_{\mathrm{SR}}\right|^{2} \geq H_{\mathrm{SR}}\right) \cdot P\left(\left|h_{\mathrm{RD}}\right|^{2} \geq H_{\mathrm{RD}}\right) \\
& =\mathrm{e}^{-H_{\mathrm{SD}}} \mathrm{e}^{-H_{\mathrm{SR}}} \mathrm{e}^{-H_{\mathrm{RD}}} .
\end{aligned}
$$

Our goal is to evaluate the maximum SE depending on the links used for TS. Therefore, we evaluate the maximum SE by varying $H_{\mathrm{SD}}, H_{\mathrm{SR}}$, and $H_{\mathrm{RD}}$ while satisfying Eq. (2). Moreover, the same values of $H_{\mathrm{SD}}, H_{\mathrm{SR}}$, and $H_{\mathrm{RD}}$ are used for all routes in the system.

\subsection{Wireless Systems with TS}

By introducing the TS, the transmission probabilities of all

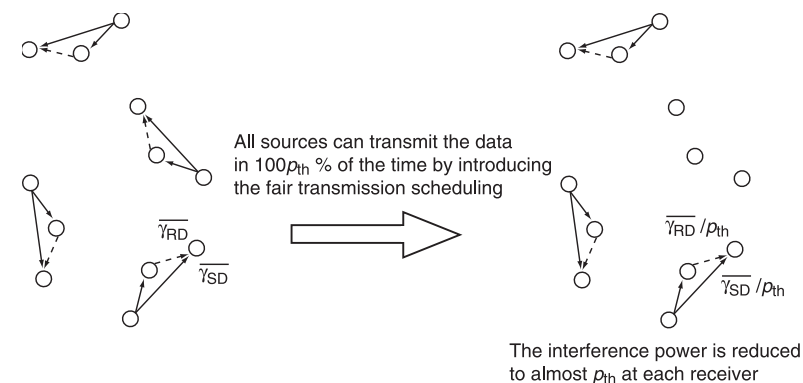

Fig. 2 Received SIR after the introduction of TS routes are reduced to $p_{\mathrm{th}}$. The number of simultaneous communications is thus reduced to $p_{\text {th }}$. The received signal-tointerference power ratio (SIR), therefore, increases to approximately $1 / p_{\text {th }}$ at each receiver, as shown in Fig. 2. The validity of this assumption will be discussed later.

\section{Total SE}

The total SE of the system is evaluated by multiplying the SE per route and the density of simultaneous communications. We use the expression given in [2] to evaluate the SE of cooperative relaying. Let us define SE as the maximum end-to-end bit rate through multiple hops per unit bandwidth. Moreover, we use the expression in [3] to evaluate the density of simultaneous communications.

\subsection{SE of Cooperative Relaying}

The source encodes the data so that all of the receivers can decode the data. The transmission rate of the source, $R_{1}$, must satisfy the following so that the relay station can decode the data:

$$
R_{1} \leq \log _{2}\left(1+\left|h_{\mathrm{SR}}\right|^{2} \overline{\gamma_{\mathrm{SR}}}\right) \triangleq R_{\text {relay }}^{\max },
$$

where $\overline{\gamma_{\mathrm{SR}}}$ is the average received SIR of the source-relay link. The transmission rate $R_{1}$ must also satisfy the following so that the destination can decode the data:

$$
R_{1} \leq \log _{2}\left(1+\left|h_{\mathrm{SD}}\right|^{2} \overline{\gamma_{\mathrm{SD}}}+\left|h_{\mathrm{RD}}\right|^{2} \overline{\gamma_{\mathrm{RD}}}\right) \triangleq R_{1}^{\max } .
$$

Here, $\overline{\gamma_{\mathrm{SD}}}$ and $\overline{\gamma_{\mathrm{RD}}}$ are the average received SIR of the source-destination link and the relay-destination link. Therefore, the SE of cooperative relaying, $R_{\text {coop }}$, is expressed as

$$
R_{\text {coop }}=\frac{1}{2} \min \left\{R_{\text {relay }}^{\max }, R_{1}^{\max }\right\},
$$

where $1 / 2$ represents the effect of dividing the available time into two time slots. Additional details are given in [2].

Let us define the $100 p \%$ outage SE as the SE that can be achieved in 100(1-p)\% of the transmission time. The $100 p \%$ outage $\mathrm{SE}, C_{\mathrm{out}}(p)$, is obtained by solving the following equation:

$$
P\left(R_{\text {coop }}<C_{\text {out }}(p)\right)=p .
$$

\subsection{Density of Simultaneous Communications}

The density of simultaneous communications is defined to be the maximum density of communications, while all stations achieve the required average SIR $\bar{\gamma}$. We consider the case in which the required average SIR is $\gamma_{\mathrm{QPSK}}$ and the density of simultaneous communications is $\rho_{\mathrm{QPSK}}$, as a standard for comparison.

We consider each distance among simultaneous communications to become $z$ times larger than the distance in 
the standard case. The interference power at each receiver is then reduced to $1 / z^{\alpha}$. Here, $\alpha$ represents the path loss exponent. Thus, the average SIR $\bar{\gamma}$ is expressed as $\bar{\gamma}=z^{\alpha} \gamma_{\mathrm{QPSK}}$. On the other hand, the density of simultaneous communications becomes $1 / z^{2}$ times smaller than that in the standard case. The density of simultaneous communications is, therefore, given by

$$
\rho(\bar{\gamma})=\frac{\rho_{\mathrm{QPSK}}}{z^{2}} \cdot p_{\mathrm{th}}=\left(\frac{\gamma_{\mathrm{QPSK}}}{\bar{\gamma}}\right)^{2 / \alpha} \rho_{\mathrm{QPSK}} \cdot p_{\mathrm{th}},
$$

where $p_{\text {th }}$ represents the average reduction of the density of simultaneous communications resulting from the introduction of the TS.

\section{Results}

\subsection{Interference Power after Introducing TS}

Computer simulation is used to examine the validity of the assumption described in Sect. 2.3 by evaluating the effect of introducing the TS on the interference power. Let the distance from the source to the destination be denoted by $D$. The simulation area is set to a $50 D \times 50 D$ square area. All sources transmit the data using cooperative relaying. The relay station is assumed to be located at the center point of the line segment from the source to the destination. The simulation parameters are listed in Table 1.

Each transmitter is assumed to be able to transmit its data if the interference power from each of the other stations is lower than a threshold. This assumption is equivalent to carrier sense [8]. This threshold is used as a parameter to change the number of simultaneous communications. When the number of simultaneous communications is increased, the average received SIR is decreased. Two time slots are randomly allocated to the source and the relay station under the condition that both of time slots can transmit the data. After no other station can begin to transmit the data, the interference power of each link is evaluated. Let the interference power at the destination at this time be denoted by $I_{0}$, and let the average received SIR of the sourcedestination link at this time be denoted by $\overline{\gamma_{\mathrm{SD}}}$. The value of $\overline{\gamma_{\mathrm{SD}}}$ is the average value for all receivers. Then, the TS is introduced so that each route transmits in $100 p_{\text {th }} \%$ of the time. The route can transmit the data when the square of the channel gain of the source-destination link is above $H_{\mathrm{th}}$, where $H_{\mathrm{th}}=-\ln \left(p_{\mathrm{th}}\right)$. Let the interference power at this time be denoted by $I_{1}$. The value of $I_{1} / I_{0}$ represents the rate of decrease in the interference power by introducing the TS.

Figure 3 shows the cumulative density function (CDF) of $I_{1} / I_{0}$. The value of $I_{1} / I_{0}$ is distributed from 0.4 to 0.6

\begin{tabular}{c|c} 
Table $1 \quad$ Parameters used in the evaluation. \\
\hline Parameters & Values \\
\hline Path loss exponent $\alpha$ & 2 \\
Channel model & Rayleigh fading \\
Area environment & Interference-limited \\
\hline
\end{tabular}

when $p_{\text {th }}=0.5$ and is distributed from 0.7 to 0.9 when $p_{\text {th }}=0.8$. This is because the number of simultaneous communications is reduced to approximately $p_{\text {th }}$ by introducing the TS. However, there is a slight difference between $I_{1} / I_{0}$ and $p_{\text {th }}$ because the stations are randomly located.

\subsection{Numerical Results}

The outage $\mathrm{SE}$ is evaluated using the parameters shown in Table 1 . We evaluate the maximum $1 \%$ outage SE, $C_{\text {out }}^{\max }(1)$, by varying $H_{\mathrm{SD}}, H_{\mathrm{SR}}$, and $H_{\mathrm{RD}}$ while satisfying Eq. (2). The $1 \%$ outage $\mathrm{SE}$ per area will be given by multiplying $C_{\text {out }}^{\max }(1)$ and the density of simultaneous communications, $\rho\left(\overline{\gamma_{\mathrm{SD}}}\right)$, i.e., $C_{\text {out }}^{\max }(1) \rho\left(\overline{\gamma_{\mathrm{SD}}}\right)=C_{\text {out }}^{\max }(1)\left(\gamma_{\mathrm{QPSK}} / \overline{\gamma_{\mathrm{SD}}}\right)^{(2 / \alpha)} \rho_{\mathrm{QPSK}} p_{\text {th }}$. Figures 4(a) and 4(b) show the $1 \%$ outage SE per area when the relay station is located at the center point of the line segment from the source to the destination $\overline{\gamma_{\mathrm{SR}}}=\overline{\gamma_{\mathrm{RD}}}=$ $\left.2^{\alpha} \overline{\gamma_{\mathrm{SD}}}\right)$. The value of $p_{\text {th }}$ is set to 0.5 in Fig. 4(a) and is set to 0.8 in Fig. 4(b). The symbols in the figures represent the links used for TS. For example, "SD, SR" means that the channel gains of the source-destination link and the sourcerelay link are used for TS and $H_{\mathrm{RD}}$ is set to zero. The case of "w/o TS" represents the $1 \%$ outage SE of the cooperative relaying system without the TS ( $p_{\text {th }}$ is set to 1 ).

The lowest $1 \%$ outage SE is achieved in the case of "SD, RD," where the source-relay link is not used for TS. This implies that the channel gain of the source-relay link must be high in order to achieve high spectral efficiency. This is because the relay station has to correctly decode the signal from the source. The highest $1 \%$ outage SE per area is achieved in the cases of "SD, SR, RD" and "SR, RD." This implies that the channel gain of the source-destination link can be ignored in TS. This is because $\overline{\gamma_{\mathrm{SD}}}$ is assumed to be low compared to $\overline{\gamma_{\mathrm{RD}}}$, and thus the source-destination link has less of an impact on Eq. (4) and almost no impact on the outage $\mathrm{SE}$. When the position of the relay station changes so that $\overline{\gamma_{\mathrm{RD}}}<\overline{\gamma_{\mathrm{SD}}}$, the source-relay link has almost no impact on the SE.

These results do not depend on $p_{\text {th }}$ because the same

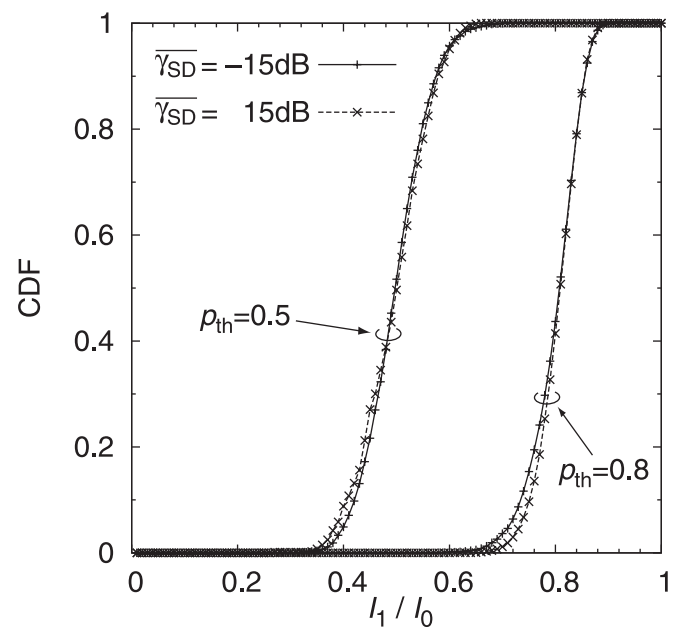

Fig. 3 Effect of introducing the TS on the average interference power. 


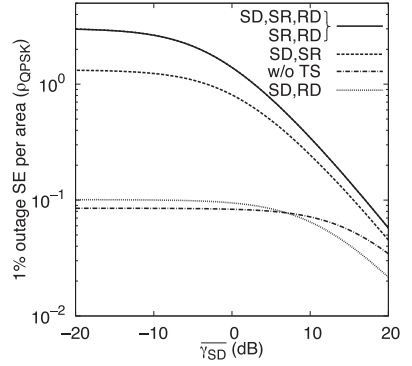

(a) $p_{\text {th }}=0.5$.

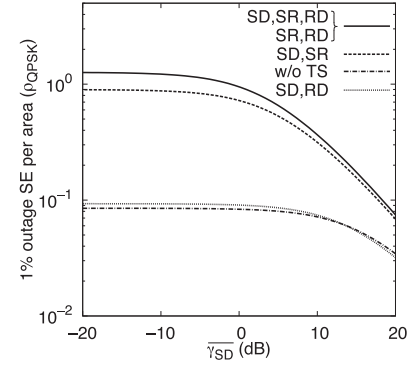

(b) $p_{\text {th }}=0.8$.
Fig. $41 \%$ outage SE per area when $\overline{\gamma_{\mathrm{SR}}}=\overline{\gamma_{\mathrm{RD}}}=2^{\alpha} \overline{\gamma_{\mathrm{SD}}}$

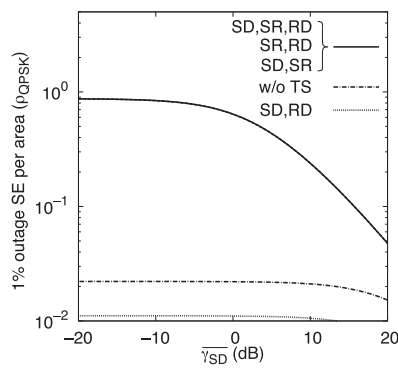

(a) $p_{\text {th }}=0.5$.

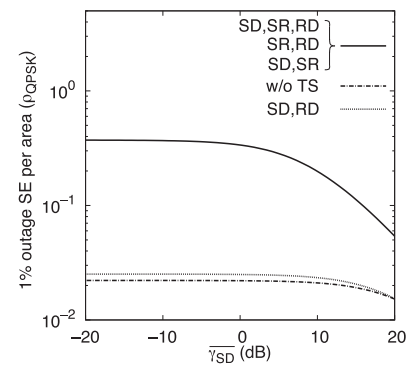

(b) $p_{\text {th }}=0.8$.
Fig. $51 \%$ outage SE per area when $\overline{\gamma_{\mathrm{SR}}}=\overline{\gamma_{\mathrm{RD}}}=\overline{\gamma_{\mathrm{SD}}}$.

distribution is used for the channel gain of each link. However, the value of the maximum $1 \%$ outage SE per area is different for Fig. 4(a) and Fig. 4(b). The 1\% outage SE in Fig. 4(b) is higher when $\overline{\gamma_{\mathrm{SD}}} \geq 10 \mathrm{~dB}$. This is because each station has a greater chance to transmit the data, and therefore the density of simultaneous communications is higher. On the other hand, the 1\% outage SE in Fig. 4(a) is higher when $\overline{\gamma_{\mathrm{SD}}}<10 \mathrm{~dB}$. This is because the interference power is decreased, and so the SIR increases at each station.

Figures 5(a) and 5(b) show the maximum 1\% outage SE per area when the source, destination, and relay station are located at the vertices of an equilateral triangle $\left(\overline{\gamma_{\mathrm{SR}}}=\right.$ $\left.\overline{\gamma_{\mathrm{RD}}}=\overline{\gamma_{\mathrm{SD}}}\right)$. The value of $p_{\text {th }}$ is set to 0.5 in Fig. 5(a) and is set to 0.8 in Fig. 5(b). The highest $1 \%$ outage SE is achieved in the cases of "SD, SR, RD," "SR, RD," and "SD, SR." This implies that when the receiver receives multiple signals with the same average received power, the channel gain of one of the signals must be high in order to achieve high spectral efficiency.

\section{Conclusion}

We evaluated the spectral efficiency of a cooperative relaying system with transmission scheduling based on the channel gains of multiple links. The channel gain of the sourcerelay link must be high because the relay has to correctly decode the signal from the source. In contrast, the channel gain of the source-destination link can be ignored for transmission scheduling when the average received power of the source-destination link is low.

\section{Acknowledgments}

The present study was supported in part by a Grant-in-Aid for Scientific Research (A) (No. 20246067) from the Japan Society for the Promotion of Science (JSPS).

\section{References}

[1] R.U. Nabar, H. Bölcskei, and F.W. Kneübuhler, "Fading relay channels: Performance limits and space-time signal design,” IEEE J. Sel. Areas Commun., vol.22, no.6, pp.1099-1109, Aug. 2004.

[2] J.N. Laneman, D.N.C. Tse, and G.W. Wornell, "Cooperative diversity in wireless networks: Efficient protocols and outage behavior," IEEE Trans. Inf. Theory, vol.50, no.12, pp.3062-3080, Dec. 2004.

[3] K. Yamamoto, H. Maruyama, T. Shimizu, H. Murata, and S. Yoshida, "Spectral efficiency of fundamental cooperative relaying in interference-limited environments," IEICE Trans. Commun., vol.E91-B, no.8, pp.2674-2682, Aug. 2008.

[4] X. Qin and R. Berry, "Exploiting multiuser diversity for medium access control in wireless networks," Proc. IEEE Infocom'03, vol.2, pp.1084-1094, April 2003.

[5] L. Dong, T. Li, and E.F. Huang, "Opportunistic transmission scheduling for multiuser MIMO systems," Proc. IEEE International Conference on Acoustics, Speech, and Signal Processing (ICASSP'03), vol.5, pp.V-65-68, April 2003.

[6] G. Kulkarni, V. Raghunathan, and M. Srivastava, "Joint end-to-end scheduling, power control and rate control in multi-hop wireless networks," Proc. IEEE GLOBECOM'04, vol.5, pp.3357-3362, Nov.Dec. 2004

[7] M.S. Alouini and A.J. Goldsmith, "Area spectral efficiency of cellular mobile radio systems," IEEE Trans. Veh. Technol., vol.48, no.4, pp.1047-1066, July 1999.

[8] IEEE Std. 802.11, "Wireless LAN medium access control (MAC) and physical layer (PHY) specifications," IEEE Std. 802.11-1999 edition, 1999. 